University of Texas at El Paso

ScholarWorks@UTEP

\title{
Towards Decision Making under Interval, Set-Valued, Fuzzy, and Z- Number Uncertainty: A Fair Price Approach
}

Joe Lorkowski

The University of Texas at El Paso, lorkowski@acm.org

Vladik Kreinovich

The University of Texas at El Paso, vladik@utep.edu

Rafik Aliev

Azerbaijan State Oil Academy, raliev@asoa.edu.az

Follow this and additional works at: https://scholarworks.utep.edu/cs_techrep

Part of the Computer Sciences Commons

Comments:

Technical Report: UTEP-CS-14-07a

Published in Proceedings of the IEEE World Congress on Computational Intelligence, Beijing, China, July 6-11, 2014.

\section{Recommended Citation}

Lorkowski, Joe; Kreinovich, Vladik; and Aliev, Rafik, "Towards Decision Making under Interval, Set-Valued, Fuzzy, and Z-Number Uncertainty: A Fair Price Approach" (2014). Departmental Technical Reports (CS). 817.

https://scholarworks.utep.edu/cs_techrep/817

This Article is brought to you for free and open access by the Computer Science at ScholarWorks@UTEP. It has been accepted for inclusion in Departmental Technical Reports (CS) by an authorized administrator of ScholarWorks@UTEP. For more information, please contact Iweber@utep.edu. 


\section{Towards Decision Making under Interval, Set-Valued, Fuzzy, and Z-Number Uncertainty: A Fair Price Approach}

\author{
Joe Lorkowski and Vladik Kreinovich \\ Department of Computer Science \\ University of Texas at El Paso \\ $500 \mathrm{~W}$. University \\ El Paso, TX 79968, USA \\ Emails: lorkowski@acm.org, vladik@utep.edu
}

\author{
Rafik Aliev \\ Azerbaijan State Oil Academy \\ Azadlig Ave. 20 \\ AZ1010, Baki, Azerbaijan \\ Email: raliev@asoa.edu.az
}

\begin{abstract}
In this paper, we explore one of the possible ways to make decisions under uncertainty: namely, we explain how to define a fair price for a participation in such a decision, and then select an alternative for which the corresponding fair price is the largest. This idea is explained on the examples of interval uncertainty, set-valued, fuzzy, and Z-number uncertainty.
\end{abstract}

\section{Formulation OF THE PROBlem}

Need for decision making. In many practical situations, we have several alternatives, and we need to select one of these alternatives. For example:

- a person saving for retirement needs to find the best way to invest money,

- a company needs to select a location for its new plant,

- a designer must select one of several possible designs for a new airplane,

- a medical doctor needs to select a treatment for a patient, etc.

Need for decision making under uncertainty. Decision making is easier if we know the exact consequences of each alternative selection.

Often, however, we only have an incomplete information about consequences of different alternatives, and we need to select an alternative under this uncertainty.

How decisions under uncertainty are made now. Traditional decision making theory mostly concentrates on the case of probabilistic uncertainty, when for each alternative $a$, we know the probability $p_{i}(a)$ of different outcomes $i$; see, e.g., [2], [6], [11]. In this case, it can be proven that preferences of a rational decision maker can be described by assigning, to each possible outcome, a value $u_{i}$ called its utility. Then, the attractiveness of each alternative to this particular decision maker can be characterized by the corresponding expected value of its utility $\bar{u}(a) \stackrel{\text { def }}{=} \sum_{i} p_{i}(a) \cdot u_{i}$ : the larger the expected utility, the better the alternative.
Often, however, we do not know these probabilities. For example, sometimes, we only know the range $[\underline{u}, \bar{u}]$ of possible utility values, but we do not know the probability of different values within this range. It has been shown that in this case, it makes sense to select an alternative for which the expression $\alpha_{H} \cdot \bar{u}+\left(1-\alpha_{H}\right) \cdot \underline{u}$ is the largest possible, where a number $\alpha_{H} \in[0,1]$ described the optimism level of a decision maker.

- When $\alpha_{H}=1$, the decision maker completely ignores the worst-case possibility $\underline{u}$ and bases his/her decision exclusively on the best-case scenario, with outcome $\bar{u}$. This case corresponds to the maximum optimism.

- When $\alpha_{H}=0$, the decision maker completely ignores the best-case possibility $\bar{u}$ and bases his/her decision exclusively on the worst-case scenario, with outcome $\underline{u}$. This case corresponds to the maximum pessimism.

Most actual decision makers take both worst-case and bestcase outcomes into account, so their decisions correspond to the values $\alpha_{H} \in(0,1)$. This idea was first proposed by the Nobelist L. Hurwicz; it is known as the Hurwicz optimismpessimism criterion [4], [6].

What if we have fuzzy uncertainty? There are many semiheuristic methods of decision making under fuzzy uncertainty, methods which have led to many practical applications; see, e.g., [5], [10]. In [1], a consistent approach to decision making under fuzzy (and more general) uncertainty is described which is based on the extension of the notion of utility to interval, fuzzy, and Z-number uncertainty.

Decision making under uncertainty: remaining problems. In spite of all these successes, there are still many practical problems in which it is not completely clear how to make an appropriate decision, so there is still a need for a consistent general methodology for decision making under uncertainty.

What we do in this paper. In this paper, we provide foundations for the new methodology of decision making under uncertainty, a methodology which is based on a natural idea of a fair price. 
Fair price approach: an idea. When we have a full information about an object, we can express our desirability of each possible situation, e.g., by declaring a price that we are willing to pay to get involved in this situation. Once these prices are set, selecting the most preferable alternative is easy: we just select the alternative for which the participation price is the highest - since this is clearly the most desirable alternative.

In decision making under uncertainty, the situation is not so clear, since it is not easy to come up with a fair price. A natural idea is to develop techniques for producing such fair prices - these prices can then be used in decision making, to select an appropriate alternative.

What we do. In this paper, we show how to describe the fair price under interval, set-valued, fuzzy, and Z-number uncertainty.

\section{CASE OF INTERVAL UNCERTAINTY}

Interval uncertainty: description. Let us start with a simple case of uncertainty, in which, instead of knowing the exact gain $u$ of selecting an alternative, we only know the lower bound $u$ and the upper bound $\bar{u}$ on this gain - and we have no information on which values from the corresponding interval $[\underline{u}, \bar{u}]$ are more probable or less probable. This situation, in which the only information that we have about the gain $u$ is that this gain belongs to the interval $[\underline{u}, \bar{u}]$, is called interval uncertainty.

Assigning fair price under interval uncertainty: description of the problem. We want to assign, to each interval $[\underline{u}, \bar{u}]$, a number $P([\underline{u}, \bar{u}])$ describing the fair price of this interval. In other words, we need a function $P([\underline{u}, \bar{u}])$ that takes an interval as an input and returns a real number. There are several reasonable requirements that this function must satisfy.

First, since in all cases, the gain is larger than or equal to $\underline{u}$ and is smaller than or equal to $\bar{u}$, it is reasonable to require that the price should also be larger than or equal to $\underline{u}$ and smaller than or equal to $\bar{u}: \underline{u} \leq P([\underline{u}, \bar{u}]) \leq \bar{u}$.

Second, if we keep the lower endpoint $\underline{u}$ intact but increase the upper bound, this means that we are keeping all the previous possibilities, but we are allowing new possibilities with a higher gain. In this case, it is reasonable to require that the corresponding price increases (or at least that it does not decrease). In other words, if $\underline{u}=\underline{v}$ and $\bar{u}<\bar{v}$, then $P([\underline{u}, \bar{u}]) \leq P([\underline{v}, \bar{v}])$.

Similar, if we dismiss some low-gain alternatives, this should increase (or at least not decrease) the fair price. So, in general, if $u \leq \underline{v}$ and $\bar{u} \leq \bar{v}$, then we should have $P([\underline{u}, \bar{u}]) \leq P([\underline{v}, \bar{v}])$

To describe the third requirement, let us consider the situation when we have two consequent decisions. Let us assume that these decisions are independent from each other, in the sense that the second decision does not depend on the first one. We can view this situation in two different ways:

- we can consider two decision processes separately, or

- we can consider a single decision process in which we select a pair of alternatives:
- the first alternative corresponding to the first decision process, and

- the second alternative corresponding to the second decision process.

If we know the exact gains $u$ and $v$ in each of the decision processes, this means that we are willing to pay:

- the amount $u$ to participate in the first process,

- the amount $v$ to participate in the second decision process, and

- the total of $u+v$ to participate in both decision processes.

In general, it is reasonable to require that even under uncertainty, the fair price $u+v$ of selecting two alternatives in the two decision processes is equal to the sum of the fair prices $u$ and $v$ of selecting each of these alternatives in the corresponding decision process.

Let us describe this requirement for the case when the consequences of each alternative are only known with interval uncertainty. About the gain $u$ from the first alternative, we only know that this (unknown) gain value belongs to the interval $[\underline{u}, \bar{u}]$. About the gain $v$ from the second alternative, we only know that this gain belongs to the interval $[\underline{v}, \bar{v}]$. The overall gain $u+v$ can thus take any value from the interval $[\underline{u}, \bar{u}]+[\underline{v}, \bar{v}]=[\underline{u}+\underline{v}, \bar{u}+\bar{v}]$. Thus, the above requirement about the fair prices takes the form

$$
P([\underline{u}+\underline{v}, \bar{u}+\bar{v}])=P([\underline{u}, \bar{u}])+P([\underline{v}, \bar{v}]) .
$$

Thus, we arrive at the following definition.

Definition 1. By $a$ fair price under interval uncertainty, we mean a function $P([\underline{u}, \bar{u}])$ that assigns, to every interval, a real number, and which satisfies the following properties:

- $\quad \underline{u} \leq P([\underline{u}, \bar{u}]) \leq \bar{u}$ for all $u$ (conservativeness);

- if $\underline{u}=\underline{v}$ and $\bar{u}<\bar{v}$, then $P([\underline{u}, \bar{u}]) \leq P([\underline{v}, \bar{v}])$ (monotonicity);

- for all $\underline{u}, \bar{u}, \underline{v}$, and $\bar{v}$, we have

$$
P([\underline{u}+\underline{v}, \bar{u}+\bar{v}])=P([\underline{u}, \bar{u}])+P([\underline{v}, \bar{v}])
$$

(additivity).

Proposition 1 [7] . Each fair price under interval uncertainty has the form

$$
P([\underline{u}, \bar{u}])=\alpha_{H} \cdot \bar{u}+\left(1-\alpha_{H}\right) \cdot \underline{u}
$$

for some real number $\alpha_{H} \in[0,1]$.

Comments.

- We thus get a new justification of the Hurwicz optimism-pessimism criterion that we described in Section I.

- We reproduce the proof from [7] since other proofs from this paper use its ideas and techniques. 


\section{Proof.}

$1^{\circ}$. Let us first consider the value $\alpha_{H} \stackrel{\text { def }}{=} P([0,1])$ corresponding to the simplest possible interval $[0,1]$. Due to conservativeness, we have $0 \leq \alpha_{H} \leq 1$.

$2^{\circ}$. Let us now compute the value $P([0, m])$ for positive integer values $m$

The interval $[0, m]$ can be represented as the sum of $m$ intervals equal to $[0,1]$ :

$$
[0, m]=[0,1]+\ldots+[0,1] \quad(m \text { times }) .
$$

Thus, due to additivity, we have

$$
\begin{gathered}
P([0, m])=P([0,1])+\ldots+P([0,1]) \quad(m \text { times })= \\
\alpha_{H}+\ldots+\alpha_{H} \quad(m \text { times })=\alpha_{H} \cdot m .
\end{gathered}
$$

$3^{\circ}$. Now, let us compute the value $z \stackrel{\text { def }}{=} P\left(\left[0, \frac{1}{n}\right]\right)$ for a positive integer $n$.

In this case, the interval $[0,1]$ can be represented as the sum of $n$ intervals equal to $\left[0, \frac{1}{n}\right]$ :

$$
[0,1]=\left[0, \frac{1}{n}\right]+\ldots+\left[0, \frac{1}{n}\right] \quad(n \text { times }) .
$$

Thus, due to additivity, we have

$$
\alpha_{H}=z+\ldots+z \quad(n \text { times }),
$$

i.e., $\alpha_{H}=z \cdot n$ and hence, $z=\alpha_{H} \cdot \frac{1}{n}$.

$4^{\circ}$. For every two positive integers $m>0$ and $n>0$, the interval $\left[0, \frac{m}{n}\right]$ can be represented as the sum of $m$ intervals equal to $\left[0, \frac{1}{n}\right]$. Thus,

$P\left(\left[0, \frac{m}{n}\right]\right)=m \cdot P\left(\left[0, \frac{1}{n}\right]\right)=m \cdot\left(\alpha_{H} \cdot \frac{1}{n}\right)=\alpha_{H} \cdot \frac{m}{n}$.

$5^{\circ}$. We have proved that for rational values $r=\frac{m}{n}$, we have $P([0, r])=\alpha_{H} \cdot r$. Let us prove that the same property $P([0, x])=\alpha_{H} \cdot x$ holds for every positive real value $x$.

To prove this property, we use monotonicity. Each real number $x$ can be approximated, with arbitrary accuracy, by two rational numbers $r<x<r^{\prime}$. Due to monotonicity, we have $P([0, r]) \leq P([0, x]) \leq P\left(\left[0, r^{\prime}\right]\right)$. Due to Part 4 of this proof, we thus conclude that $\alpha_{H} \cdot r \leq P([0, x]) \leq \alpha_{H} \cdot r^{\prime}$. When $r \rightarrow x$ and $r^{\prime} \rightarrow x$, we get $\alpha_{H} \cdot r \rightarrow \alpha_{H} \cdot x$ and $\alpha_{H} \cdot r^{\prime} \rightarrow \alpha_{H} \cdot x$ and thus, $P([0, x])=\alpha_{H} \cdot x$.

$6^{\circ}$. Now, we are ready to prove the proposition. For each $u$ and $\bar{u}$, we have $[\underline{u}, \bar{u}]=[\underline{u}, \underline{u}]+[0, \bar{u}-\underline{u}]$. Thus, due to additivity,

$$
P([\underline{u}, \bar{u}])=P([\underline{u}, \underline{u}])+P([0, \bar{u}-\underline{u}]) .
$$

For the first term, due to conservativeness, we have $\underline{u} \leq$ $P([\underline{u}, \underline{u}]) \leq \underline{u}$ and thus, $P([\underline{u}, \underline{u}])=\underline{u}$. For the second term, due to Part 5 of this proof, we get $P([0, \bar{u}-\underline{u}])=\alpha_{H} \cdot(\bar{u}-\underline{u})$. Thus, the above additivity formula leads to

$$
P([\underline{u}, \bar{u}])=\underline{u}+\alpha_{H} \cdot(\bar{u}-\underline{u}),
$$

which is exactly $\alpha_{H} \cdot \bar{u}+\left(1-\alpha_{H}\right) \cdot \underline{u}$. The proposition is proven.

\section{CASE OF SET-VALUED UNCERTAINTY}

Description of the case. In some cases, in addition to knowing that the actual gain belongs to the interval $[\underline{u}, \bar{u}]$, we also know that some values from this interval cannot be possible values of this gain. For example, if we buy an obscure lottery ticket for a simple prize-or-no-prize lottery from a remote country, we either get the prize or lose the money. In this case, the set of possible values of the gain consists of two values.

In a closer-to-home lottery, we usually have an additional information about the outcomes that we can take into account when making a decision: e.g., we usually know the probability of a prize (or prizes, if there are different prizes). However, for an obscure lottery, it is reasonable to imagine that we have no additional information. In this case, the only information that we have is the (2-element) set of possible outcomes.

In general, instead of a (bounded) interval of possible values, we can consider a more general bounded set of possible values. It makes sense to consider bounded sets $S$ that contain all their limits points. Indeed, if $x_{n} \in S$ for all $n$ and $x_{n} \rightarrow x$, then, for any given accuracy, $x$ is undistinguishable from some possible value $x_{n}$-thus, in effect, the value $x$ itself is possible. Such sets are known as closed sets. So, in this section, we will consider bounded closed sets.

Assigning fair price under set-valued uncertainty: description of the problem. We want to assign, to each bounded closed set $S$, a number $P(S)$ describing the fair price of this set. In other words, we need a function $P(S)$ that takes a set as an input and returns a real number. There are several reasonable requirements that this function must satisfy.

First, for the case when the set $S$ is an interval, we must get the fair price as described by Proposition 1.

Second, if we have two independent alternatives described by sets $S$ and $S^{\prime}$, then we should have $P\left(S+S^{\prime}\right)=P(S)+$ $P\left(S^{\prime}\right)$, where

$$
S+S^{\prime} \stackrel{\text { def }}{=}\left\{x+x^{\prime}: x \in S \text { and } x^{\prime} \in S^{\prime}\right\}
$$

is the set of all possible sums $x+x^{\prime}$.

Thus, we arrive at the following definition.

Definition 2. By a fair price under set-valued uncertainty, we mean a function $P(S)$ that assigns, to every bounded closed set $S$, a real number, and which satisfies the following properties:

- $\quad$ a restriction of this function to intervals $S=[\underline{u}, \bar{u}]$ is a fair price under interval uncertainty - in the sense of Definition 1 (conservativeness);

- for every two sets $S$ and $S^{\prime}$, we have $P\left(S+S^{\prime}\right)=$ $P(S)+P\left(S^{\prime}\right)$ (additivity). 
Proposition 2. Each fair price under interval uncertainty has the form $P([\underline{u}, \bar{u}])=\alpha_{H} \cdot \sup S+\left(1-\alpha_{H}\right) \cdot \inf S$ for some real number $\alpha_{H} \in[0,1]$.

Proof. Due to conservativeness, for intervals $S=[\underline{u}, \bar{u}]$ ), the function $P(S)$ is a fair price under interval uncertainty and thus, due to Proposition 1 , has the form $P([\underline{u}, \bar{u}])=\alpha_{H} \cdot \bar{u}+$ $\left(1-\alpha_{H}\right) \cdot \underline{u}$.

For each bounded set $S$, its infimum $\underline{s} \stackrel{\text { def }}{=} \inf S$ and its supremum $\bar{s} \stackrel{\text { def }}{=} \sup S$ are finite. By definition, inf $S$ is a lower bound (it is the greatest lower bound) and $\sup S$ is an upper bound (it is the least upper bound); thus, we have $S \subseteq[\underline{s}, \bar{s}]$. Both inf $S$ and $\sup S$ are limits of points from the set $S$; since the set $S$ is closed, it contains these limits: $\{\underline{s}, \bar{s}\} \subseteq S \subseteq[\underline{s}, \bar{s}]$.

Let us prove that $[\underline{s}, \bar{s}]+S=[2 \underline{s}, 2 \bar{s}]$. Indeed, from the definition (6) of set addition, one can easily conclude that if $S^{\prime} \subseteq S^{\prime \prime}$, then $S+S^{\prime} \subseteq S+S^{\prime \prime}$. In particular, $\{\underline{s}, \bar{s}\} \subseteq S \subseteq$ $[\underline{s}, \bar{s}]$ implies that

$$
[\underline{s}, \bar{s}]+\{\underline{s}, \bar{s}\} \subseteq[\underline{s}, \bar{s}]+S \subseteq[\underline{s}, \bar{s}]+[\underline{s}, \bar{s}] .
$$

Here, $[\underline{s}, \bar{s}]+\{\underline{s}, \bar{s}\}=[2 \underline{s}, 2 \bar{s}]$ and similarly $[\underline{s}, \bar{s}]+[\underline{s}, \bar{s}]=$ $[2 \underline{s}, 2 \bar{s}]$. Thus,

$$
[2 \underline{s}, 2 \bar{s}] \subseteq[\underline{s}, \bar{s}]+S \subseteq[2 \underline{s}, 2 \bar{s}]
$$

and so, indeed, $[\underline{s}, \bar{s}]+S=[2 \underline{s}, 2 \bar{s}]$.

Now, additivity implies that $P(S)=P([2 \underline{s}, 2 \bar{s}])-$ $P([\underline{s}, \bar{s}])$. Substituting the expression $P([\underline{u}, \bar{u}])=\alpha_{H} \cdot \bar{u}+$ $\left(1-\alpha_{H}\right) \cdot \underline{u}$ for the fair price of intervals into this formula, we get the desired expression for $P(S)$. The proposition is proven.

\section{IV. (CRISP) Z-NuMBERS, Z-INTERVALS, AND Z-SETS: CASEs When the Probabilities ARE CRISP}

Description of the case. In the previous sections, we assumed that we are $100 \%$ certain that the actual gain is contained in the given interval (or set). In reality, mistakes are possible, so usually, we are only certain that $u$ belongs to the corresponding interval or set with some probability $0<p<1$. In such situations, to fully describe our knowledge, we need to describe both the interval (or set) and this probability $p$.

In the general context, after supplementing the information about a quantity with the information of how certain we are about this piece of information, we get what L. Zadeh calls a Z-number [13]. Because of this:

- we will call a pair consisting of a (crisp) number and a (crisp) probability a crisp Z-number;

- we will call a pair consisting of an interval and a probability a Z-interval; and

- we will call a pair consisting of a set and a probability a Z-set.

In this section, we will describe fair prices for crisp Z-numbers, Z-intervals, and Z-sets for situations when the probability $p$ is known exactly.

Operations on the corresponding pairs. When we have two independent sequential decisions, and we are $100 \%$ sure that the first decision leads to gain $u$ and the second decision leads to gain $v$, then, as we have mentioned earlier, the user's total gain is equal to the sum $u+v$. In this section, we consider the situation in which:

- for the first decision, our degree of confidence in the gain estimate $u$ is described by some probability $p$;

- for the second decision, our degree of confidence in the gain estimate $v$ is described by some probability $q$.

The estimate $u+v$ is valid only if both gain estimates are correct. Since these estimates are independent, the probability that they are both correct is equal to the product $p \cdot q$ of the corresponding probabilities. Thus:

- $\quad$ for crisp Z-numbers $(u, p)$ and $(v, q)$, the sum is equal to $(u+v, p \cdot q)$;

- for Z-intervals $([\underline{u}, \bar{u}], p)$ and $[\underline{v}, \bar{v}], q)$, the sum is equal to $([\underline{u}+\underline{v}, \bar{u}+\bar{v}], p \cdot q)$;

- $\quad$ finally, for Z-sets $(S, p)$ and $\left(S^{\prime}, q\right)$, the sum is equal to $\left(S+S^{\prime}, p \cdot q\right)$.

Let us analyze these cases one by one.

Case of crisp Z-numbers. Since the probability $p$ is usually known with some uncertainty, it makes sense to require that the fair price of a crisp Z-number $(u, p)$ continuously depend on $p$, so that small changes in $p$ lead to small changes in the fair price - and the closer our estimate to the actual value of the probability, the closer the estimated fair price should be to the actual fair price.

Thus, we arrive at the following definitions.

Definition 3. By a crisp Z-number, we mean a pair $(u, p)$ of two real numbers such that $0<p \leq 1$.

Definition 4. By a fair price under crisp Z-number uncertainty, we mean a function $P(u, p)$ that assigns, to every crisp Znumber, a real number, and which satisfies the following properties:

- $\quad P(u, 1)=u$ for all $u$ (conservativeness);

- for all $u, v, p$, and $q$, we have $P(u+v, p \cdot q)=$ $P(u, p)+P(v, q)$ (additivity);

- the function $P(u, p)$ is continuous in $p$ (continuity).

Proposition 3. Each fair price under crisp Z-number uncertainty has the form $P(u, p)=u-k \cdot \ln (p)$ for some real number $k$.

\section{Proof.}

$1^{\circ}$. By additivity, we have $P(u, p)=P(u, 1)+P(0, p)$. By conservativeness, we have $P(u, 1)=u$; thus, $P(u, p)=u+$ $P(0, p)$. So, it is clear that to find the general expression for the fair price function $P(u, p)$, it is sufficient to find the values $P(0, p)$ corresponding to $u=0$.

$2^{\circ}$. Additivity implies that $P(0, p \cdot q)=P(0, p)+P(0, q)$. 
$3^{\circ}$. Let us first consider the value $p=e^{-1}$ which corresponds to $\ln (p)=-1$. The corresponding value of $P(0, p)$ will be denoted by $k \stackrel{\text { def }}{=} P\left(0, e^{-1}\right)$. Then, for $p=e^{-1}$, we have the desired expression $P(0, p)=-k \cdot \ln (p)$.

$4^{\circ}$. Let us now consider the values $P\left(0, e^{-m}\right)$ for positive integer values $m$. The probability $e^{-m}$ can be represented as a product of $m$ values $e^{-1}$ :

$$
e^{-m}=e^{-1} \cdot \ldots \cdot e^{-1} \quad(m \text { times }) .
$$

Thus, due to additivity, we have

$$
\begin{gathered}
P\left(0, e^{-m}\right)=P\left(0, e^{-1}\right)+\ldots+P\left(0, e^{-1}\right) \quad(m \text { times })= \\
m \cdot k .
\end{gathered}
$$

Since for $p=e^{-m}$, we have $\ln (p)=-m$, we thus have $P(0, p)=-k \cdot \ln (p)$ for these values $p$.

$5^{\circ}$. Now, let us estimate the value $P(0, p)$ for $p=e^{-1 / n}$, for a positive integer $n$.

In this case, the value $e^{-1}$ can be represented as a product of $n$ probabilities equal to $e^{-1 / n}$ :

$$
e^{-1}=e^{-1 / n} \cdot \ldots \cdot e^{-1 / n} \quad(n \text { times }) .
$$

Thus, due to additivity, we have

$k=P\left(0, e^{-1}\right)=P\left(0, e^{-1 / n}\right)+\ldots+P\left(0, e^{-1 / n}\right) \quad(n$ times $)$, i.e.,

$$
k=n \cdot P\left(0, e^{-1 / n}\right)
$$

and hence, $P\left(0, e^{-1 / n}\right)=\frac{k}{n}$. Therefore, for $p=e^{-1 / n}$, we also have $P(0, p)=-k \cdot \ln (p)$.

$6^{\circ}$. For every two positive numbers $m>0$ and $n>0$, the probability $e^{-m / n}$ can be represented as the product of $m$ probabilities equal to $e^{-1 / n}$. Thus, due to additivity, we have $P\left(0, e^{-m / n}\right)=m \cdot P\left(0, e^{-1 / n}\right)=k \cdot \frac{m}{n}$. Hence, for the values $p=e^{-m / n}$ for which the logarithm $\ln (p)$ is a rational number, we have $P(0, p)=-k \cdot \ln (p)$.

$7^{\circ}$. Every real number $\ell \stackrel{\text { def }}{=} \ln (p)$ can be approximated, with arbitrary accuracy, by rational numbers $\ell_{n} \rightarrow \ell$ for which $p_{n} \stackrel{\text { def }}{=} e^{-\ell_{n}} \rightarrow e^{-\ell}=p$. For these rational numbers, we have $P\left(0, p_{n}\right)=-k \cdot \ln \left(p_{n}\right)$. Thus, when $n \rightarrow \infty$ and $p_{n} \rightarrow p$, by continuity, we have $P(0, p)=-k \cdot \ln (p)$.

From Part 1, we know that $P(u, p)=u+P(0, p)$; thus, indeed, $P(u, p)=u-k \cdot \ln (p)$. The proposition is proven.

Cases of Z-intervals and Z-sets. Similar results hold for Zintervals and Z-sets; in both results, we will use the fact that we already know how to set a fair price for the case when $p=1$.

Definition 5. By a Z-interval, we mean a pair $([\underline{u}, \bar{u}], p)$ consisting of an interval $[\underline{u}, \bar{u}]$ and a real numbers $p$ such that $0<p \leq 1$.

Definition 6. By a fair price under Z-interval uncertainty, we mean a function $P([\underline{u}, \bar{u}], p)$ that assigns, to every Z-interval, a real number, and which satisfies the following properties:
- for some $\alpha_{H} \in[0,1]$ and for all $\underline{u} \leq \bar{u}$, we have $P([\underline{u}, \bar{u}], 1)=\alpha_{H} \cdot \bar{u}+\left(1-\alpha_{H}\right) \cdot \underline{u}$ (conservativeness);

- $\quad$ for all $\underline{u}, \bar{u}, \underline{v}, \bar{v}, p$, and $q$, we have

$$
\begin{aligned}
& P([\underline{u}+\underline{v}, \bar{u}+\bar{v}], p \cdot q)= \\
& P([\underline{u}, \bar{u}], p)+P([\underline{v}, \bar{v}], q)
\end{aligned}
$$

(additivity).

Proposition 4. Each fair price under Z-interval uncertainty has the form $P([\underline{u}, \bar{u}], p)=\alpha_{H} \cdot \bar{u}+\left(1-\alpha_{H}\right) \cdot \underline{u}-k \cdot \ln (p)$ for some real numbers $\alpha_{H} \in[0,1]$ and $k$.

Proof. By additivity, we have $P([\underline{u}, \bar{u}], p)=P([\underline{u}, \bar{u}], 1)+$ $P(0, p)$. By conservativeness, we have

$$
P([\underline{u}, \bar{u}], 1)=\alpha_{H} \cdot \bar{u}+\left(1-\alpha_{H}\right) \cdot \underline{u} .
$$

For $P(0, p)$, similarly to the proof of Proposition 3 , we conclude that $P(0, p)=-k \cdot \ln (p)$ for some real number $k$. The proposition is proven.

Definition 7. By a Z-set, we mean a pair $(S, p)$ consisting of a closed bounded set $S$ and a real numbers $p$ such that $0<p \leq 1$.

Definition 8. By a fair price under Z-set-valued uncertainty, we mean a function $P(S, p)$ that assigns, to every Z-interval, a real number, and which satisfies the following properties:

- for some $\alpha_{H} \in[0,1]$ and for all sets $S$, we have

$$
P(S, 1)=\alpha_{H} \cdot \sup S+\left(1-\alpha_{H}\right) \cdot \inf S
$$

(conservativeness);

- for all $S, S^{\prime}, p$, and $q$, we have $P\left(S+S^{\prime}, p \cdot q\right)=$ $P(S, p)+P\left(S^{\prime}, q\right)$ (additivity).

Proposition 5. Each fair price under Z-set-valued uncertainty has the form

$$
P(S, p)=\alpha_{H} \cdot \sup S+\left(1-\alpha_{H}\right) \cdot \inf S-k \cdot \ln (p)
$$

for some real numbers $\alpha_{H} \in[0,1]$ and $k$.

Proof. By additivity, we have $P(S, p)=P(S, 1)+P(\{0\}, p)$. By conservativeness, we have

$$
P(S, 1)=\alpha_{H} \cdot \sup S+\left(1-\alpha_{H}\right) \cdot \inf S .
$$

For $P(\{0\}, p)$, similarly to the proof of Proposition 3, we conclude that $P(\{0\}, p)=-k \cdot \ln (p)$ for some real number $k$. The proposition is proven. 


\section{V. (CRISP) Z-NuMBERS, Z-INTERVALS, AND Z-SETS: Cases When Probabilities ARe KNOWN With INTERVAL OR SET-VALUED UNCERTAINTY}

Motivations. When we know the exact probabilities $p$ and $q$ that the corresponding estimates are correct, then the probability that both estimates are correct is equal to the product $p \cdot q$.

Similarly to the fact that we often do not know the exact gain, we often do not know the exact probability $p$. Instead, we may only know the interval $[p, \bar{p}]$ of possible values of $p$, or, more generally, a set $\mathcal{P}$ of possible values of $p$. If we know $p$ and $q$ with such uncertainty, what can we then conclude about the product $p \cdot q$ ?

For positive values $p$ and $q$, the function $p \cdot q$ is increasing as a function of both variables: if we increase $p$ and/or increase $q$, the product increases. Thus, if the only information that we have the probability $p$ is that this probability belongs to the interval $[p, \bar{p}]$, and the only information that we have the probability $q$ is that this probability belongs to the interval $[\underline{q}, \bar{q}]$, then:

- the smallest possible value of $p \cdot q$ is equal to the product $\underline{p} \cdot \underline{q}$ of the smallest values;

- $\quad$ the largest possible value of $p \cdot q$ is equal to the product $\bar{p} \cdot \bar{q}$ of the largest values; and

- $\quad$ the set of all possible values $p \cdot q$ is the interval

$$
[\underline{p} \cdot \underline{q}, \bar{p} \cdot \bar{q}] \cdot
$$

For sets $\mathcal{P}$ and $\mathcal{Q}$, the set of possible values $p \cdot q$ is the set

$$
\mathcal{P} \cdot \mathcal{Q} \stackrel{\text { def }}{=}\{p \cdot q: p \in \mathcal{P} \text { and } q \in \mathcal{Q}\} .
$$

Let us find the fair price under such uncertainty.

Case of crisp Z-numbers. Let us start with the case of crisp Z-numbers under such uncertainty.

Definition 9. By $a$ crisp Z-number under interval $p$-uncertainty, we mean a pair $(u,[p, \bar{p}])$ consisting of a real number $u$ and an interval $[\underline{p}, \bar{p}] \subseteq \overline{(} 0,1]$.

Definition 10. By a fair price under crisp Z-number $p$-interval uncertainty, we mean a function $P(u,[p, \bar{p}])$ that assigns, to every crisp Z-number under interval p-uncertainty, a real number, and which satisfies the following properties:

- $\quad$ for some real number $k$, we have

$$
P(u,[p, p])=u-k \cdot \ln (p)
$$

for all $u$ and $p$ (conservativeness);

- $\quad$ for all $u, v, \underline{p}, \bar{p}, \underline{q}$, and $\bar{q}$, we have

$$
\begin{gathered}
P(u+v,[\underline{p} \cdot \underline{q}, \bar{p}, \bar{q}])= \\
P(u,[\underline{p}, \bar{p}])+P(v,[\underline{q}, \bar{q}])
\end{gathered}
$$

(additivity);

- the function $P(u,[\underline{p}, \bar{p}])$ is continuous in $\underline{p}$ and $\bar{p}$ (continuity).
Proposition 6. Each fair price under crisp Z-number p-interval uncertainty has the form

$$
P(u,[\underline{p}, \bar{p}])=u-(k-\beta) \cdot \ln (\bar{p})-\beta \cdot \ln (\underline{p})
$$

for some real numbers $k$ and $\beta \in[0,1]$.

\section{Proof.}

$1^{\circ}$. By additivity, we have $P(u,[\underline{p}, \bar{p}])=P(u, \bar{p})+$ $P(0,[p, 1])$, where $p \stackrel{\text { def }}{=} p / \bar{p}$. By conservativeness, we know that $P(u, \bar{p})=u-k \cdot \ln (\overline{\bar{p}})$. Thus, $P(u, p)=u-k \cdot \ln (\bar{p})+$ $P(0,[p, 1])$. So, to find the general expression for the fair price function $P(u,[p, \bar{p}])$, it is sufficient to find the values $P(0,[p, 1])$ corresponding to $u=0$ and $\bar{p}=1$.

$2^{\circ}$. For the values $P(0,[p, 1])$, additivity implies that

$$
P(0,[p \cdot q, 1])=P(0,[p, 1])+P(0,[q, 1]) .
$$

In Parts 2 of the proof of Proposition 3, we had a similar property for a continuous function $P(0, p)$, and we proved, in Parts 2-6 of that proof, that this property implies that this continuous function is equal to $-c \cdot \ln (p)$ for some real number $c$. Thus, we can similarly conclude that

$$
P(0,[p, 1])=-\beta \cdot \ln (p)
$$

for some real number $\beta$.

$3^{\circ}$. From Part 1 of this proof, we know that

$$
P(u,[\underline{p}, \bar{p}])=u-k \cdot \ln (\bar{p})+P(0,[p, 1]) ;
$$

thus,

$$
P(u,[\underline{p}, \bar{p}])=u-k \cdot \ln (\bar{p})-\beta \cdot \ln (p) .
$$

Substituting $p=p / \bar{p}$ into this formula and taking into account that $\ln (p)=\ln (\underline{p})-\ln (\bar{p})$, we get the desired formula.

Definition 11. By a crisp Z-number under set-valued $p$ uncertainty, we mean a pair $(u, \mathcal{P})$ consisting of a real number $u$ and $a$ bounded closed set $\mathcal{P} \subseteq(0,1]$.

Comment. One can easily show that for each closed set $\mathcal{P} \subseteq$ $(0,1]$, we have $\inf \mathcal{P}>0$.

Definition 12. By $a$ fair price under crisp Z-number $p$-setvalued uncertainty, we mean a function $P(u, \mathcal{P})$ that assigns, to every crisp Z-number under set-valued p-uncertainty, a real number, and which satisfies the following properties:

- $\quad$ for some real numbers $k$ and $\beta$, we have

$$
P(u,[\underline{p}, \bar{p}])=u-(k-\beta) \cdot \ln (\bar{p})-\beta \cdot \ln (\underline{p})
$$

for all $u, p$, and $\bar{p}$ (conservativeness);

- for all $u, v, \mathcal{P}$, and $\mathcal{Q}$, we have

$$
P(u+v, \mathcal{P} \cdot \mathcal{Q})=P(u, \mathcal{P})+P(v, \mathcal{Q})
$$

(additivity). 
Proposition 7. Each fair price under crisp Z-number p-setvalued uncertainty has the form

$$
P(u, \mathcal{P})=u-(k-\beta) \cdot \ln (\sup \mathcal{P})-\beta \cdot \ln (\inf \mathcal{P})
$$

for some real number $\beta \in[0,1]$.

Proof. By additivity, we have $P(u, \mathcal{P})=P(u,\{1\})+P(0, \mathcal{P})$, i.e., due to conservativeness, $P(u, \mathcal{P})=u+P(0, \mathcal{P})$. So, to find the expression for $P(u, \mathcal{P})$, it is sufficient to find the values $P(0, \mathcal{P})$. Similarly to prove of Proposition 2 , we can prove that

$$
\mathcal{P} \cdot[\inf \mathcal{P}, \sup \mathcal{P}]=\left[(\inf \mathcal{P})^{2},(\sup \mathcal{P})^{2}\right] .
$$

Due to additivity, this implies that

$$
\begin{gathered}
P\left(0,\left[(\inf \mathcal{P})^{2},(\sup \mathcal{P})^{2}\right]\right)= \\
P(0, \mathcal{P})+P(0,[\inf \mathcal{P}, \sup \mathcal{P}]),
\end{gathered}
$$

hence

$$
\begin{gathered}
P(0, \mathcal{P})= \\
P\left(0,\left[(\inf \mathcal{P})^{2},(\sup \mathcal{P})^{2}\right]\right)-P(0,[\inf \mathcal{P}, \sup \mathcal{P}]) .
\end{gathered}
$$

Due to conservativeness, we know the values in the righthand side of this equality. Substituting these values, we get the desired formula.

Case of $\mathbf{Z}$-intervals and $\mathbf{Z}$-sets. Let us extend the above results to Z-sets (and to their particular case: Z-intervals).

Definition 13. By a Z-set under set-valued $p$-uncertainty, we mean a pair $(S, \mathcal{P})$ consisting of a bounded closed set $S$ and a bounded closed set $\mathcal{P} \subseteq(0,1]$.

Definition 14. By $a$ fair price under Z-set $p$-set-valued uncertainty, we mean a function $P(S, \mathcal{P})$ that assigns, to every $Z$-set under set-valued p-uncertainty, a real number, and which satisfies the following properties:

- for some real number $\alpha_{H} \in[0,1]$, we have

$$
P(S, 1)=\alpha_{H} \cdot \sup S+\left(1-\alpha_{H}\right) \cdot \inf S
$$

for all $S$ (conservativeness);

- $\quad$ for some real numbers $k$ and $\beta$, we have

$$
P(u, \mathcal{P})=u-(k-\beta) \cdot \ln (\sup \mathcal{P})-\beta \cdot \ln (\inf \mathcal{P})
$$

for all $u$ and $\mathcal{P}$ (conservativeness);

- $\quad$ for all $S, S^{\prime}, \mathcal{P}$, and $\mathcal{Q}$, we have

$$
P\left(S+S^{\prime}, \mathcal{P} \cdot \mathcal{Q}\right)=P(S, \mathcal{P})+P(Q, \mathcal{Q})
$$

(additivity).

Proposition 8. Each fair price under Z-set p-set-valued uncertainty has the form

$$
\begin{gathered}
P(S, \mathcal{P})=\alpha_{H} \cdot \sup S+\left(1-\alpha_{H}\right) \cdot \inf S- \\
(k-\beta) \cdot \ln (\bar{p})-\beta \cdot \ln (\underline{p}) .
\end{gathered}
$$

\section{CASE OF FUZZY AND Z-NUMBER UNCERTAINTY}

Fuzzy numbers: reminder. In the above text, we first considered situations when about each value of gain $u$, the expert is either absolutely sure that this value is possible or absolutely sure that this value is not possible. Then, we took into account the possibility that the expert is not $100 \%$ certain about that - but we assumed that the expert either knows the exact probability $p$ describing his/her degree of certainty, or that the expert is absolutely sure which probabilities can describe his/her uncertainty and which cannot.

In reality, an expert is often uncertain about the possible values, and uncertain about possible degrees of uncertainty. To take this uncertainty into account, L. Zadeh introduced the notion of a fuzzy set [5], [10], [12], where, to each possible value of $u$, we assign a degree $\mu(u) \in[0,1]$ to which this value $u$ is possible. Similarly, a fuzzy set $\mu_{p}:[0,1] \rightarrow[0,1]$ can describe the degrees to which different probability values are possible.

In this paper, we restrict ourselves to fuzzy numbers $s$, i.e., fuzzy sets for which the membership function is different from 0 only on a bounded set, where it first monotonically increases until it reaches a point $\bar{s}$ at which $\mu(\bar{s})=1$, and then monotonically decreases from 1 to 0 .

Operations on fuzzy numbers. Operations on fuzzy numbers are usually described in terms of Zadeh's extension principle: if two quantities $u$ and $v$ are described by membership functions $\mu_{1}(u)$ and $\mu_{2}(v)$, then their sum $w=u+v$ is described by the membership function $\mu(w)=\max _{u, v: u+v=w} \min \left(\mu_{1}(u), \mu_{2}(v)\right)$, and their product $w=$ $u \cdot v$ is described by the membership function $\mu(w)=$ $\max _{u, v: u \cdot v=w} \min \left(\mu_{1}(u), \mu_{2}(v)\right)$.

It is known that these operations can be equivalently described in terms of the $\alpha$-cuts. An $\alpha$-cut of a fuzzy number $\mu(u)$ is defined as an interval $\mathbf{u}(\alpha)=\left[u^{-}(\alpha), u^{+}(\alpha)\right]$, where

$$
\begin{gathered}
u^{-}(\alpha) \stackrel{\text { def }}{=} \inf \{u: \mu(u) \geq \alpha\} \text { and } \\
u^{+}(\alpha) \stackrel{\text { def }}{=} \sup \{u: \mu(u) \geq \alpha\} .
\end{gathered}
$$

The $\alpha$-cuts corresponding to the sum $w=u+v$ can be described, for every $\alpha$, as

$$
\left[w^{-}(\alpha), w^{+}(\alpha)\right]=\left[u^{-}(\alpha), u^{+}(\alpha)\right]+\left[v^{-}(\alpha), v^{+}(\alpha)\right],
$$

or, equivalently, as

$$
\left[w^{-}(\alpha), w^{+}(\alpha)\right]=\left[u^{-}(\alpha)+v^{-}(\alpha), u^{+}(\alpha)+v^{+}(\alpha)\right] .
$$

Similarly, the $\alpha$-cuts corresponding to the product $w=u \cdot v$ can be described as

$$
\left[w^{-}(\alpha), w^{+}(\alpha)\right]=\left[u^{-}(\alpha), u^{+}(\alpha)\right] \cdot\left[v^{-}(\alpha), v^{+}(\alpha)\right] .
$$

If both fuzzy numbers $u$ and $v$ are non-negative (e.g., if they are limited to the interval $[0,1])$, then the $\alpha$-cuts corresponding to the product can be described as

$$
\left[w^{-}(\alpha), w^{+}(\alpha)\right]=\left[u^{-}(\alpha) \cdot v^{-}(\alpha), u^{+}(\alpha) \cdot v^{+}(\alpha)\right] .
$$

Fair price of fuzzy numbers. Let us start with describing the fair price of fuzzy numbers. Similarly to the interval case, 
a natural requirement is monotonicity: if for all $\alpha$, we have $s^{-}(\alpha) \leq t^{-}(\alpha)$ and $s^{+}(\alpha) \leq t^{+}(\alpha)$, then the fair price of $t$ should be larger than or equal to the fair price of $s$. It is also reasonable to require continuity: that small changes in $\mu(u)$ should lead to small changes in the fair price.

Definition 15. By a fair price under fuzzy uncertainty, we mean a function $P(s)$ that assigns, to every fuzzy number $s$, a real number, and which satisfies the following properties:

- if a fuzzy number $s$ is located between $\underline{u}$ and $\bar{u}$, then $\underline{u} \leq P(s) \leq \bar{u}$ (conservativeness);

- $\quad$ if a fuzzy number $w$ is the sum of fuzzy numbers $u$ and $v$, then we have $P(w)=P(u)+P(v)$ (additivity);

- $\quad$ if for all $\alpha$, we have

$$
s^{-}(\alpha) \leq t^{-}(\alpha) \text { and } s^{+}(\alpha) \leq t^{+}(\alpha),
$$

then we have $P(s) \leq P(t)$ (monotonicity);

- $\quad$ if a sequence of membership functions $\mu_{n}$ uniformly converges to $\mu$, then we should have $P\left(\mu_{n}\right) \rightarrow P(\mu)$ (continuity).

Riemann-Stieltjes integral: reminder. We will see, the fair price of a fuzzy number is described in terms of a RiemannStieltjes integral. For readers who need a reminder of what this integral is, a brief reminder is presented as an appendix.

Proposition 9. For a fuzzy number $s$ with a continuous membership function $\mu(x), \alpha$-cuts $\left[s^{-}(\alpha), s^{+}(\alpha)\right]$ and a point $s_{0}$ at which $\mu\left(s_{0}\right)=1$, the fair price is equal to

$$
P(s)=s_{0}+\int_{0}^{1} k^{-}(\alpha) d s^{-}(\alpha)-\int_{0}^{1} k^{+}(\alpha) d s^{+}(\alpha),
$$

for appropriate functions $k^{-}(\alpha)$ and $k^{+}(\alpha)$.

Discussion. When the function $g(x)$ is differentiable, the Riemann-Stieltjes integral $\int_{a}^{b} f(x) d g(x)$ is equal to the usual integral

$$
\int_{a}^{b} f(x) \cdot g^{\prime}(x) d x
$$

where $g^{\prime}(x)$ denotes the derivative. When the function $f(x)$ is also differentiable, we can use integration by part and get yet another equivalent form

$$
f(b) \cdot g(b)-f(a) \cdot g(a)+\int_{a}^{b} F(x) \cdot g(x) d x,
$$

with $F(x)=-f^{\prime}(x)$. In general, a Stieltjes integral can be represented in a similar form for some generalized function $F(x)$ (see, e.g., [3]; generalized function are also known as distributions; we do not use this term to avoid confusion with probability distributions). Thus, the above general formula can be described as

$$
P(s)=\int_{0}^{1} K^{-}(\alpha) \cdot s^{-}(\alpha) d \alpha+\int_{0}^{1} K^{+}(\alpha) \cdot s^{+}(\alpha) d \alpha
$$

for appropriate generalized functions $K^{-}(\alpha)$ and $K^{+}(\alpha)$.
Conservativeness means that for a crisp number located at $s_{0}$, we should have $P(s)=s_{0}$. For the above formula, this means that

$$
\int_{0}^{1} K^{-}(\alpha) d \alpha+\int_{0}^{1} K^{+}(\alpha) d \alpha=1 .
$$

For a fuzzy number which is equal to the interval $[\underline{u}, \bar{u}]$, the above formula leads to

$$
P(s)=\left(\int_{0}^{1} K^{-}(\alpha) d \alpha\right) \cdot \underline{u}+\left(\int_{0}^{1} K^{+}(\alpha) d \alpha\right) \cdot \bar{u} .
$$

Thus, Hurwicz optimism-pessimism coefficient $\alpha_{H}$ is equal to $\int_{0}^{1} K^{+}(\alpha) d \alpha$. In this sense, the above formula is a generalization of Hurwicz's formula to the fuzzy case.

\section{Proof.}

$1^{\circ}$. For every two real numbers $u \geq 0$ and $\gamma \in[0,1]$, let us define a fuzzy number $s_{\gamma, u}(x)$ with the following membership function: $\mu_{\gamma, u}(0)=1, \mu_{\gamma, u}(x)=\gamma$ for $x \in(0, u]$, and $\mu_{\gamma, u}(x)=0$ for all other $x$. For this fuzzy numbers, $\alpha$-cuts have the following form: $\mathbf{s}_{\gamma, u}(\alpha)=[0,0]$ for $\alpha>\gamma$, and $\mathbf{s}_{\gamma, u}(\alpha)=[0, u]$ for $\alpha \leq \gamma$.

Based on the $\alpha$-cuts, one can easily check that $s_{\gamma, u+v}=$ $s_{\gamma, u}+s_{\gamma, v}$. Thus, due to additivity, $P\left(s_{\gamma, u+v}\right)=P\left(s_{\gamma, u}\right)+$ $P\left(s_{\gamma, v}\right)$. Due to monotonicity, the value $P\left(s_{\gamma, u}\right)$ monotonically depends on $u$. Thus, similarly to the proof of Proposition 1 , we can conclude that $P\left(s_{\gamma, u}\right)=k^{+}(\gamma) \cdot u$ for some value $k^{+}(\gamma)$

By definition, the fuzzy number $s_{\gamma, u}$ is located between 0 and $u$, so, due to conservativeness, we have $0 \leq P\left(s_{\gamma, u}\right) \leq u$ for all $u$. This implies that $0 \leq k^{+}(\gamma) \leq 1$.

$2^{\circ}$. Let us now consider a fuzzy number $s$ whose membership function is equal to 0 for $x<0$, jumps to 1 for $x=0$, and then continuously decrease to 0 . For this fuzzy number, all $\alpha$ cuts have the form $\left[0, s^{+}(\alpha)\right]$ for some $s^{+}(\alpha)$. By definition of an $\alpha$-cut, the value $s^{+}(\alpha)$ decreases with $\alpha$.

For each sequence of values

$$
\alpha_{0}=1<\alpha_{1}<\alpha_{2}<\ldots<\alpha_{n-1}<\alpha_{n}=1,
$$

we can define a fuzzy number $s_{n}$ with the following $\alpha$-cuts:

- $s_{n}^{-}(\alpha)=0$ for all $\alpha$; and

- $\quad$ when $\alpha \in\left[\alpha_{i}, \alpha_{i+1}\right)$, then $s_{n}^{+}(\alpha)=s^{+}\left(\alpha_{i}\right)$.

Since the membership function of $s$ is continuous, when $\max \left(\alpha_{i+1}-\alpha_{i}\right) \rightarrow 0$, we have $s_{n} \rightarrow s$, and thus, $P\left(s_{n}\right) \rightarrow$ $P(s)$.

One can check that the fuzzy number $s_{n}$ can be represented as a sum of $n$ fuzzy numbers

$$
\begin{gathered}
s_{n}=s_{\alpha_{n-1}, s^{+}\left(\alpha_{n-1}\right)}+s_{\alpha_{n-2}, s^{+}\left(\alpha_{n-2}\right)-s^{+}\left(\alpha_{n-1}\right)}+\ldots+ \\
s_{\alpha_{1}, \alpha_{1}-\alpha_{2} .} .
\end{gathered}
$$

Thus, due to additivity, we have

$$
\begin{gathered}
P\left(s_{n}\right)=P\left(s_{\alpha_{n-1}, s^{+}\left(\alpha_{n-1}\right.}\right)+ \\
P\left(s_{\alpha_{n-2}, s^{+}\left(\alpha_{n-2}\right)-s^{+}\left(\alpha_{n-1}\right)}\right)+\ldots+P\left(s_{\alpha_{1}, \alpha_{1}-\alpha_{2}}\right) .
\end{gathered}
$$


Substituting the expression for $P\left(s_{\gamma, u}\right)$ from Part 1 of this proof, we conclude that

$$
\begin{gathered}
P\left(s_{n}\right)=k^{+}\left(\alpha_{n-1}\right) \cdot s^{+}\left(\alpha_{n-1}\right)+ \\
k^{+}\left(\alpha_{n-2}\right) \cdot\left(s^{+}\left(\alpha_{n-2}\right)-s^{+}\left(\alpha_{n-1}\right)\right)+\ldots+ \\
k^{+}\left(\alpha_{1}\right) \cdot\left(\alpha_{1}-\alpha_{2}\right) .
\end{gathered}
$$

The right-hand side is minus the integral sum for the RiemannStieltjes integral $\int_{0}^{1} k^{+}(\gamma) d s^{+}(\gamma)$. Since we have $P\left(s_{n}\right) \rightarrow$ $P(s)$, this means that the integral sums always converges, the Riemann-Stieltjes integral is defined, and the limit $P(s)$ is equal to this integral.

$3^{\circ}$. Similarly, for fuzzy numbers $s$ whose membership function $\mu(x)$ continuously increases from 0 to 1 as $x$ increases to 0 and is equal to 0 for $x>0$, the $\alpha$-cuts are equal to $\left[s^{-}(\alpha), 0\right]$, and $P(s)=\int_{0}^{1} k^{-}(\gamma) d s^{-}(\gamma)$ for an appropriate function $k^{-}(\gamma)$.

$4^{\circ}$. A general fuzzy number $g$, with $\alpha$-cuts $\left[g^{-}(\alpha), g^{+}(\alpha)\right]$ and a point $g_{0}$ at which $\mu\left(g_{0}\right)=1$, can be represented as the sum of three fuzzy numbers:

- a crisp number $g_{0}$;

- $\quad$ a fuzzy number whose $\alpha$-cuts are equal to

$$
\left[0, g^{+}(\alpha)-g_{0}\right] ; \text { and }
$$

- $\quad$ a fuzzy number whose $\alpha$-cuts are equal to

$$
\left[g_{0}-g^{-}(\alpha), 0\right] \text {. }
$$

By conservativeness, the fair price of the crisp number is equal to $g_{0}$. The fair prices of the second and the their fuzzy numbers can be obtained by using the formulas from Parts 2 and 3 of this proof. By additivity, the fair price of the sum is equal to the sum of the prices. By taking into account that for every constant $g_{0}, d\left(g(x)-g_{0}\right)=d g(x)$ and thus,

$$
\int f(x) d\left(g(x)-g_{0}\right)=\int f(x) d g(x),
$$

we get the desired expression.

Case of Z-number uncertainty. In this case, we have two fuzzy numbers: the fuzzy number $s$ which describes the values and the fuzzy number $p$ which describes our degree of confidence in the piece of information described by $s$.

Definition 16. By $a$ fair price under Z-number uncertainty, we mean a function $P(s, p)$ that assigns, to every pair of two fuzzy numbers $s$ and $p$ such that $p$ is located on an interval $\left[p_{0}, 1\right]$ for some $p_{0}>0$, a real number, and which satisfies the following properties:

- $\quad$ if a fuzzy number $s$ is located between $\underline{u}$ and $\bar{u}$, then $\underline{u} \leq P(s, 1) \leq \bar{u}$ (conservativeness);

- $\quad$ if $w=u+v$ and $r=p \cdot q$, then

$$
P(w, r)=P(u, p)+P(v, q)
$$

(additivity);

- if for all $\alpha$, we have

$$
s^{-}(\alpha) \leq t^{-}(\alpha) \text { and } s^{+}(\alpha) \leq t^{+}(\alpha),
$$

then we have $P(s, 1) \leq P(t, 1)$ (monotonicity);

- $\quad$ if $s_{n} \rightarrow s$ and $p_{n} \rightarrow p$, then $P\left(s_{n}, p_{n}\right) \rightarrow P(p, s)$ (continuity).

Proposition 10. For a fuzzy number $s$ with $\alpha$-cuts $\left[s^{-}(\alpha), s^{+}(\alpha)\right]$ and a fuzzy number $p$ with $\alpha$-cuts $\left[p^{-}(\alpha), p^{+}(\alpha)\right]$, we have

$$
\begin{aligned}
& P(s, p)=\int_{0}^{1} K^{-}(\alpha) \cdot s^{-}(\alpha) d \alpha+\int_{0}^{1} K^{+}(\alpha) \cdot s^{+}(\alpha) d \alpha+ \\
& \int_{0}^{1} L^{-}(\alpha) \cdot \ln \left(p^{-}(\alpha)\right) d \alpha+\int_{0}^{1} L^{+}(\alpha) \cdot \ln \left(p^{+}(\alpha)\right) d \alpha
\end{aligned}
$$

for appropriate generalized functions $K^{ \pm}(\alpha)$ and $L^{ \pm}(\alpha)$.

Proof. Due to additivity, we have

$$
P(s, p)=P(s, 1)+P(0, p) .
$$

We already know the expression for $P(s, 1)$; we thus need to find the expression for $P(0, p)$. For logarithms, we have $\ln (p \cdot q)=\ln (p)+\ln (q)$, so in terms of logarithms, additivity takes the usual form

$$
P(0, \ln (p)+\ln (q))=P(0, \ln (p))+P(0, \ln (q)) .
$$

Thus, similarly to the proof of Proposition 9, we conclude that

$$
\begin{gathered}
P(0, p)=\int_{0}^{1} L^{-}(\alpha) \cdot \ln \left(p^{-}(\alpha)\right) d \alpha+ \\
\int_{0}^{1} L^{+}(\alpha) \cdot \ln \left(p^{+}(\alpha)\right) d \alpha .
\end{gathered}
$$

By applying additivity to this expression and to the known expression for $P(s, 1)$, we get the desired formula.

\section{CONCLUSIONS AND REMAINING PROBLEMS}

Fair price approach to decision making under uncertainty. In many practical situations, we need to select an alternative when do not know the exact consequences of each possible selection.

For example, instead of knowing the exact resulting gain $u$, we may only know the bounds $\underline{u}$ and $\bar{u}$ for which $\underline{u} \leq u \leq \bar{u}$ (case of interval uncertainty).

We may also know, e.g., that the gain will be somewhat larger than a certain value $u_{0}$; such an uncertainty can be naturally described by a fuzzy set.

To gauge the attractiveness of each alternative under such an alternative, we propose to estimate the fair price corresponding to each uncertainty. We show that for many types of uncertainty, natural common-sense requirements lead to explicit formulas for the fair price.

For interval uncertainty, the resulting formula coincides with the known Hurwicz optimism-pessimism criterion. We also provide explicit formulas for the cases of fuzzy and Znumber uncertainty.

Need for practical applications and practical testing of the fair price idea. So far, our work has been mainly theoretical. 
We would like to see it applied to practical decision making problems - and to gauge how well the decision makers will be satisfied with the resulting recommendations.

Need for further generalizations. In this paper, we described how to define fair price when we have one piece of information: a fuzzy set $S$ of gains with a fuzzy set $\mathcal{P}$ describing how confident we are in $S$. In practice, we may have several such pieces of information. It is desirable to come up with formulas which describe fair price under such multiple pieces of information - formulas which are uniquely determined by additivity and similar reasonable conditions.

Another open question is how to extend the above formulas for fair price to the case of interval-valued and, more general, type-2 fuzzy sets; see, e.g., [8], [9].

\section{ACKNOWLEDGMENTS}

This work was supported in part by the National Science Foundation grants HRD-0734825 and HRD-1242122 (CyberShARE Center of Excellence) and DUE-0926721, by Grants 1 T36 GM078000-01 and 1R43TR000173-01 from the National Institutes of Health, and by a grant N62909-12-1-7039 from the Office of Naval Research.

The authors are thankful to the anonymous referees for valuable suggestions.

\section{REFERENCES}

[1] R. A. Aliev, Fundamentals of the Fuzzy Logic-Based Generalized Theory of Decisions, Springer Verlag, Berlin, Heidelberg, 2013.

[2] P. C. Fishburn, Nonlinear Preference and Utility Theory, John Hopkins Press, Baltimore, Maryland, 1988

[3] I. M. Gelfand, G. E. Shilov, and N. Ya. Vilenkin, Generalized Functions, Academic Press, New York, 1964.

[4] L. Hurwicz, Optimality Criteria for Decision Making Under Ignorance, Cowles Commission Discussion Paper, Statistics, No. 370, 1951.

[5] G. Klir and B. Yuan, "Fuzzy Sets and Fuzzy Logic", Prentice Hall, Upper Saddle River, New Jersey, 1995.
[6] R. D. Luce and R. Raiffa, Games and Decisions: Introduction and Critical Survey, Dover, New York, 1989.

[7] J. McKee, J. Lorkowski, and T. Ngamsantivong, "Note on Fair Price under Interval Uncertainty", Journal of Uncertain Systems, 2014, Vol. 8, to appear.

[8] J. M. Mendel, Uncertain Rule-Based Fuzzy Logic Systems: Introduction and New Directions, Prentice-Hall, Upper Saddle River, 2001.

[9] J. M. Mendel and D. Wu, Perceptual Computing: Aiding People in Making Subjective Judgments, IEEE Press and Wiley, New York, 2010.

[10] H. T. Nguyen and E. A. Walker, A First Course in Fuzzy Logic, Chapman and Hall/CRC, Boca Raton, Florida, 2006.

[11] H. Raiffa, Decision Analysis, McGraw-Hill, Columbus, Ohio, 1997.

[12] L. A. Zadeh, "Fuzzy sets", Information and Control, 1965, Vol. 8, pp. $338-353$.

[13] L. A. Zadeh, "A Note on Z-Numbers", Information Sciences, 2011, Vol. 181, pp. 2923-2932.

\section{APPENDIX: RIEMANN-STIELTJES INTEGRAL - REMINDER}

Riemann-Stieltjes integral is a natural generalization of the usual (Riemann) integral.

In general, an intuitive meaning of a Riemann integral $\int_{a}^{b} f(x) d x$ is that it is an area under the curve $y=f(x)$. To compute this integral, we select points

$$
a=x_{1}<x_{2}<\ldots<x_{n-1}<x_{n}=b,
$$

and approximate the curve by a piece-wise constant function $\widetilde{f}(x)=f\left(x_{i}\right)$ for $x \in\left[x_{i}, x_{i+1}\right)$. The subgraph of this piecewise constant function is a union of several rectangles, so its area is equal to the sum of the areas of these rectangles $\sum f\left(x_{i}\right) \cdot\left(x_{i+1}-x_{i}\right)$. This sum is known as the integral sum for the integral $\int_{a}^{b} f(x) d x$. Riemann's integral can be formally defined as a limit of such integral sums when $\max \left(x_{i+1}-x_{i}\right) \rightarrow 0$.

A Riemann-Stieltjes integral $\int_{a}^{b} f(x) d g(x)$ is similarly defined as the limit of the sums $\sum f\left(x_{i}\right) \cdot\left(g\left(x_{i+1}\right)-g\left(x_{i}\right)\right)$ when $\max \left(x_{i+1}-x_{i}\right) \rightarrow 0$. 